\title{
AUTOMATIZACIÓN CONTABLE Y EL FUTURO DE LA PROFESIÓN CONTABLE
}

\section{ARTÍCULO ORIGINAL}

SUMAR, Ramiro Rodrigues ${ }^{1}$

SUMAR, Ramiro Rodrigues. Automatización contable y el futuro de la profesión contable. Revista Científica Multidisciplinar Núcleo do Conhecimento. Año 06, Ed. 06, Vol. 17, págs. 167 y 181. Junio de 2021. ISSN: 2448-0959, Enlace de acceso: https://www.nucleodoconhecimento.com.br/contabilidad/profesion-contable, DOI: 10.32749/nucleodoconhecimento.com.br/contabilidad/profesion-contable

\section{RESUMEN}

La presencia de calidad en los servicios prestados es, en efecto, indispensable para la supervivencia de la profesión contable, ya que se traduce en el objetivo de un contable. Los sistemas de información, las nuevas tecnologías de inteligencia artificial y la innovación, cuando se conectan con la experiencia de los profesionales de la contabilidad, pueden resultar en un mayor rendimiento de las empresas y, en consecuencia, de la economía. Considerando que la profesión del contador busca proporcionar información tributaria a las autoridades tributarias, este artículo tiene como objetivo demostrar que esta profesión se encuentra en un proceso de migración con respecto a la automatización de tareas, con el fin de convertirse en una herramienta de gestión capaz de ayudar en la toma de decisiones asertivas, con el fin de contribuir al desempeño de la responsabilidad social de las empresas. La problematización de este estudio se basa en la siguiente pregunta problemática: ¿Cuál será el papel del contador con la automatización de la contabilidad? ¿Terminará la profesión contable como predijo el Instituto Sapiens en la investigación de 2018? Para la consecución de los objetivos, se utilizó la revisión bibliográfica. Como

\footnotetext{
${ }^{1}$ Máster universitario en Contraloría, Especialista en Gestión Tecnológica, Administración Estratégica, Seguridad de la Información y Licenciado en Ciencias Contables.
} 
resultado, el artículo demostró el papel del contador en el proceso de desarrollo y crecimiento de las empresas antes y después de la automatización de los procesos burocráticos, así como la perspectiva de futuro de la profesión. Así, se encontró que la profesión contable sufre un cambio repentino de posicionamiento para que sobreviva como profesión y mantenga su papel con la sociedad y economía del país.

Palabras clave: Automatización Contable, Contabilidad Profesional, Responsabilidad Social, Toma de Decisiones.

\section{INTRODUCCIÓN}

Hay un escenario de grandes cambios en el mercado contable, con nuevas tecnologías, uso de inteligencia artificial y automatización de procesos. Y junto a estos cambios están las preocupaciones, ansiedades e incertidumbres que el nuevo despierta en las personas (SOUZA et al., 2018).

Estos cambios en el país son vistos y sentidos por todos, donde la inflación ya no se considera un problema, las tasas de desempleo han disminuido, el acceso al crédito $y$ al consumo ha aumentado $y$, como consecuencia, las tasas de pobreza han disminuido (CUNHA, 2019).

Por lo tanto, existe un escenario empresarial, en el que cada día surgen nuevas empresas y se solicitan más profesionales, pero algunas profesiones corren el riesgo de no existir debido a la tecnología y la automatización (LEITE, 2017; ARAÚJO, 2020).

Según Rozenbaum (2018), un estudio realizado en 2018 por el instituto francés Sapiens revela que cinco profesiones están en riesgo de extinguirse, entre ellas se encuentra el profesional contable que trabaja en una oficina sin acceso a tecnología, como el uso de ordenadores.

El contador moderno, todavía tiene que lidiar con la parte burocrática, las legalidades y la gran cantidad de roles que ocupan espacio. Dado que la automatización de procesos y otros advenimientos para facilitar el trabajo contable se derivan de las tecnologías, el profesional de la contabilidad tendrá que adaptarse al uso de la 
tecnología a su favor, o ser reacio a cambiar y ver su profesión desaparecer antes de la automatización (DINIZ, 2014).

Según el estudio del instituto francés, más de dos millones de personas "tienen grandes posibilidades de ver desaparecer sus puestos de trabajo en los próximos años". Los datos para el estudio fueron de DARES (Servicio de Estudios Estadísticos del Ministerio de Trabajo de Francia) y en él, por ejemplo, se puede observar que los trabajadores bancarios pueden desaparecer por completo en 2050 y los contables en 2056 (ROZENBAUM, 2018).

La competitividad expuesta en el mercado, con emprendedores que buscan cada día innovación y nuevas tecnologías para expandir su negocio, hace que el profesional de la contabilidad se inserte en este entorno. Las empresas que se enfrentan al mercado competitivo no pueden dejar de contar junto con la ayuda del contador, ya que este profesional tiene en su atribución el objetivo de la calidad y la eficiencia en todas las áreas del negocio (CHIAVENATO, 2004).

Otro punto es que los millennials se están apoderando del mercado laboral y ganando posiciones en la jerarquía de las empresas y en la toma de decisiones. Por lo tanto, para el profesional de la contabilidad es importante conocer y entender a este público que espera agilidad y calidad en los servicios de contabilidad.

Sin embargo, el propósito de este artículo es estudiar el futuro de la profesión contable y la automatización que se ha venido produciendo en el trabajo contable. Estos cambios son necesarios para mantener al contador en el mercado competitivo, así como su importancia en la generación de nuevos puestos de trabajo, su capacidad para emprender e innovar, así como la importancia social antes de su función contable y fiscal.

La profesión contable está pasando por grandes transformaciones, de dejar de ser el profesional que solo aporta problemas y cuentas, y actuar como soporte en la gestión de las empresas. Para ello, el profesional debe adaptarse a las nuevas exigencias e 
innovar, con el fin de mantener su papel e importancia, no solo para las empresas y directivos, sino también para la sociedad, tras su papel social.

La metodología utilizada en la redacción de este artículo fue la investigación bibliográfica, a través de annais, investigación, libros, revistas, entrevistas en Internet, artículos en Internet y artículos académicos publicados. Para Gil (2008), la investigación bibliográfica se desarrolla a partir de material ya elaborado y compuesto principalmente por monografías, disertaciones, artículos científicos y libros.

Cuando se trata de propósito, Marconi y Lakatos (1992) explican que la investigación bibliográfica hace que el observador tenga la oportunidad de contactar directamente con los materiales escritos sobre el tema de la investigación y asistir al investigador en su análisis o en el momento de la manipulación de la información. La investigación bibliográfica se considera como el primer paso hacia el inicio de la investigación científica.

\section{REFERENCIA TEÓRICA}

\subsection{HISTORIA DEL PROFESIONAL DE LAS ESTADÍSTICAS}

Hay informes de que la figura del contador ha estado presente desde el comienzo de la civilización. Según ludícibus y Marion (2006, p. 32): "[...] de los pueblos más primitivos, la Contabilidad ya existía debido a la necesidad de controlar, medir y preservar los bienes familiares e incluso, debido al intercambio de bienes para una mayor satisfacción de las personas [...]".

A pesar de los informes, la existencia oficial de la contabilidad se marcó en la edad moderna, en los siglos 15 a 16, en la región de Italia. En el período del renacimiento, la profesión contable ganó espacio en áreas como: economía, artes, ciencias y tecnologías (FIGUEIREDO; STRASSBURG, 2008).

En Brasil, incluso antes de que surgieran las primeras escuelas técnicas comerciales, la contabilidad era ejercida por la figura del "contable", un profesional definido según 
el Código de Comercio de 1850 que, según Francisco D'Auria (1949), era responsable y registró las transacciones de establecimientos comerciales en ese momento (CARVALHO, 2017).

El desarrollo de la profesión contable siguió a la aprobación del Decreto-Ley № 9.295 de 27 de mayo de 1946, que creó el Consejo Federal de Contabilidad y definió las atribuciones de contadores, técnicos contables y contadores (DOS SANTOS et al., 2019).

Con el consejo la profesión se expandió y surgieron muchos problemas con la equiparación de profesionales con diferentes niveles de educación y conocimiento. $\mathrm{Si}$, por un lado, está el contador formado con un curso de pregrado, un profesional sin ningún estudio coexistió en la misma profesión (NETO, 2010).

Esto provocó el desinterés de los jóvenes por la contabilidad, ya que la situación de esta profesión no era la misma que la de otras formaciones como Derecho y Economía. ¿Cómo despertar el interés por una profesión que presenta niveles de profesionales opuestos entre sí? Esto sólo fue posible con la prueba de la competencia y la separación del técnico contable al propio contador.

En 1972, la auditoría fue regulada por la Resolución № 220, así como las Circulares № 178 y № 179 del Banco Central del Brasil. Impuso estándares demostrativos que requerían que los profesionales fueran adaptados a una auditoría por personas certificadas (ABREU, 2013; CORDERO, 2011).

Sin embargo, la profesión contable no recibió el estatus hasta diciembre de 1976 con la promulgación de la Ley 6.404 conocida como la Ley de SA (Sociedades Anônimas - Sociedad Anonima). Esta ley originó la Comisión Brasileña de Valores y Bolsa (CVM), que reemplazó al Banco Central, con respecto a la emisión de opiniones y parte de la legislación contable, así como el control del mercado de capitales en Brasil (CARVALHOSA, 2017).

Para Santos (2008, p. 9) 
[...] en los últimos años, especialmente después del final de la era de alta inflación, desde mediados de la década de 1990, ha sufrido un período de grandes cambios y adaptaciones. Las empresas que fueron más ágiles en ajustar su enfoque a su negocio principal, su negocio principal, y que ya no administraron las ganancias e ingresos inflacionarios, lograron una mayor tasa de éxito. Y, en este momento, el profesional de la contabilidad tiene una participación primaria al proporcionar orientación fiscal, corporativa y financiera. [...]. El contador, cada vez más, es la brújula de la empresa.

Se observa que las habilidades y requisitos de habilidades del profesional de la contabilidad se centran en las estrategias y la toma de decisiones en las empresas. Debido a los cambios tecnológicos, los gobiernos, las empresas y los gerentes se encuentran en un alto grado de innovación, exigiendo cada vez más al profesional de la contabilidad que, a su vez, ayuda a la toma de decisiones (DE OLIVEIRA REIS et al., 2015).

En un entorno altamente competitivo y ágil, la obtención de información de forma segura es un diferencial. La precisión y la fiabilidad también son requeridas por las empresas modernas que quieren asegurar su supervivencia en el mercado y hacer de la figura contable un científico de datos de riqueza y asientos contables, lo que le exige saber cómo interpretar los datos y delinear estrategias y caminos que garanticen mejores resultados. Todas estas demandas vienen en contra de lo que Santos (2008, p. 10) escribe en su libro:

[...] el contador no debe perder de cuenta que la contabilidad no es para él, es para el usuario. La contabilidad es el lenguaje de los negocios y este lenguaje cuenta la historia de cada empresa. Perfeccionar este lenguaje buscando aumentar su poder de predicción y utilidad para el usuario es quizás la misión principal del contador. [...]

Por lo tanto, el profesional debe estar en el proceso de aprendizaje, actualización y educación para ser un experto en su papel y en la obtención de datos que ayudan a la corporación en su objetivo social. El fácil acceso a los cursos de postgrado en el área de contabilidad brinda al individuo la oportunidad de posicionarse junto al mercado para contribuir a las empresas, la economía y la sociedad.

\subsection{DESARROLLO ECONÓMICO Y CRECIMIENTO}


Furtado (1964) define el desarrollo económico como "un proceso de cambio social mediante el cual se satisface un número creciente de necesidades humanas a través de una diferenciación en el sistema productivo resultante de la introducción de innovaciones tecnológicas".

Para Hewlett (1981), el desarrollo económico se define como "un aumento significativo en el ingreso real per cápita de una nación, con el propósito de obtener mejores alimentos, salud, educación, mejores condiciones de vida y una gama cada vez más amplia de oportunidades de trabajo y ocio para la gente de esta nación".

Por otro lado, está el crecimiento económico que, según Siedenberg (2006), consiste en un "proceso de cambios predominantemente cuantitativos, es decir, un aumento de tamaño, volumen y / o cantidad". Resumiendo lo que concluyó Vasconcellos (2000) al señalar que el crecimiento económico es el crecimiento continuo del ingreso per cápita a lo largo del tiempo.

El crecimiento y mantenimiento del producto interno bruto (PIB) promueve una mayor calidad de vida de la población. Además, las innovaciones tecnológicas, el aumento de los ingresos nacionales, la expansión de la fuerza laboral y el ahorro son otros factores que ayudan al crecimiento económico de un país.

Se observa que cuando un país muestra crecimiento económico el número de personas desempleadas disminuye, mientras que los impuestos recaudados aumentan. En este contexto, es necesario invertir más para mantener el crecimiento continuo de la economía. Mendes (2015) explica que la economía y el crecimiento de los padres han tenido un rendimiento inferior debido a algunos factores, como la educación y la alta carga tributaria.

Alineado con el crecimiento económico viene el desarrollo económico que para existir es necesario que el país mantenga indicadores y tenga factores para mejorar la calidad de vida y los ingresos de la población a lo largo del tiempo. Para Rocha (2004) lo que demuestra estos factores es: el crecimiento del bienestar económico, medido a través de indicadores económicos, entre los que es válido mencionar el producto 
nacional total y el producto nacional per cápita; reducir los niveles de pobreza, desempleo y vida social; y el aumento de las condiciones de salud, nutrición, educación y vivienda.

El Índice de Desarrollo Humano - IDH, de las Naciones Unidas (ONU), es una unidad de medida creada y utilizada para verificar el grado de desarrollo de un país determinado, que abarca salud, educación e ingresos. El índice es una referencia numérica que tiene definida su variación entre 0 y 1 , en sus definiciones, cuanto más cercano esté el valor a 1, mejor debe ser el desarrollo de esa ubicación en los ítems presentados. Ningún país tiene un IDH definido en cero o incluso en uno (FILGUEIRA et al., 2010).

Según el informe de la ONU que tiene la evaluación de 189 países, Brasil alcanzó los 0,761 puntos en 2018 , lo que representa un aumento significativo en comparación con 2013, donde recibió 0,752 .

La posición de Brasil ocupa el puesto 79 en el ranking, junto a Colombia y se ubica entre los países de alta categoría de desarrollo humano. En el informe el indicador más alto es el de Noruega con 0,954 puntos, y el peor índice de desarrollo humano es Níger con 0,377 (LEUSIN JÚNIOR, 2015).

\subsection{NUEVAS TECNOLOGÍAS EN CONTABILIDAD}

Las nuevas tecnologías terminan generando cambios estructurales en las organizaciones con su aplicación, impactando en los costos de las empresas y modificando sus procesos productivos, en vista de la competitividad en el mercado (MAT, 2010).

Scott (2009) enfatiza que el uso de la tecnología en la contabilidad es un cambio sin precedentes, es decir, no es fácil identificar si sus impactos fueron negativos o positivos. Desde otro punto de vista, Alsharayri (2011) y Choe (2004) muestran que el nivel de tecnología e información producida tiene impactos positivos. 
La tecnología tiene impactos positivos tanto en la agilidad y fiabilidad como en la seguridad de los sistemas de información utilizados en las empresas por las oficinas de contabilidad y los profesionales. La función de la información generada por los sistemas, según Allahyari y Ramazani (2011), es ayudar a sus usuarios a tomar decisiones, considerando que la contabilidad es capaz de producir información relevante para la formulación de procesos empresariales y estratégicos, para el control de actividades y para el crecimiento de las empresas.

Para Scott (2009), la ventaja de la tecnología radica en el uso de varias herramientas para acelerar la ejecución de las funciones realizadas por el contador. En este aspecto, la tecnología contribuye al avance operativo de la contabilidad frente a la competitividad, dada la rapidez y eficiencia que ofrecen los avances tecnológicos.

Acevedo (2012) muestra que si las comunicaciones en las empresas de contabilidad son rápidas pueden ayudar a aumentar la productividad, así como pueden mejorar la toma de decisiones y facilitar el crecimiento del negocio.

También según Scott (2009), una desventaja de la tecnología es su dependencia de los seres humanos. La tecnología surge de la innovación humana, por lo tanto, es un producto que repite los mismos errores humanos. Pero esto se puede evitar con procesos más robustos y con una especialización y educación más enfocadas en la automatización. También demuestra que otra desventaja es la dependencia de la profesión de la tecnología, pero ya se sabe que los estudios demuestran que el contador que existía para poner en práctica los cálculos se extinguirá, lo que choca con este pensamiento erróneo del autor.

Aribaba et al. (2011), a su vez, destacan la importancia que la tecnología añade a la sociedad, especialmente en las pequeñas empresas de servicios, dada la mejora en el rendimiento y el mayor desarrollo del negocio. Así, con más rendimiento surgen más oportunidades y ganancias, con ello se generan empleos y se beneficia la economía. 
La competitividad requiere que las organizaciones contables sean capaces de crear valor para sus clientes. Estos clientes que son contribuyentes y contratistas del personal. Según Simons (1987), al diferenciarse de sus competidores, implementar y formular una estrategia de negocio bien definida, estas organizaciones añadirán más resultados. En la estrategia, el uso de tecnologías añadidas a los sistemas contables es esencial, ya que proporciona información segura y útil (JERMIAS; GANI; 2004).

Tener una estrategia bien definida y consolidada, basada en inversiones en tecnología y calificación de personas, según Grande et al. (2011), traerá ventajas productivas y cambios favorables para sus clientes en comparación con sus competidores.

\subsection{PAPEL DEL CONTABLE EN LA INNOVACIÓN Y AUTOMATIZACIÓN DE LOS PROCESOS CONTABLES}

El número de oficinas contables aumenta y contribuye a la disminución del desempleo, entre los años 2009 a 2012, según Gondim (2014). Hubo un aumento en más del $60 \%$ de los empleos formales y del $5 \%$ en las nuevas firmas de contabilidad.

El Consejo Federal de Contabilidad (CFC, 2014), presenta, en su portal, una investigación realizada que demuestra que más del $58 \%$ de los profesionales de la contabilidad tienen la preferencia de trabajar en empresas del sector privado o abrir su propia oficina para tener que licitar e ingresar al sector público. Ver que estos datos muestran la contribución a la generación de números de empleo formal e informal, ya que el profesional contable trabaja con los gerentes de las empresas (sus clientes) colaborando para que el negocio evolucione y crezca y necesite más empleados.

Al ayudar a los empresarios o invertir en su propio negocio, utilizando los conocimientos prácticos y teóricos adquiridos, el contador tiene un papel importante en la economía. En los últimos años la contabilidad ha dejado de utilizar sólo una calculadora y resmas de papel (NOGUEIRA, 2010).

Ver que Coelho (2015) explica que el contador pasó de ser la persona que registra hechos pasados a ser un consultor estratégico con una visión prospectiva y amplia del 
mercado. A partir de estos cambios el contactor tiene la misión de automatizar procesos, generar más rendimiento y comcomworking datos estadísticos con el fin de apoyar a las empresas y directivos en la toma de decisiones.

Fávero et al. (2006) muestran que "la contabilidad tiene como objetivo generar información para los diversos usuarios para que puedan tomar decisiones". Este objetivo parece sencillo, pero los factores subjetivos que intervienen en él dificultan este proceso, la dificultad de entender los datos y el distanciamiento del contable del gestor dificultan su cumplimiento. Veamos que hay variables para una buena comunicación, y estas deben ser estudiadas y la especialidad del contador, para que se logre su objetivo, como profesional contable.

Como público objetivo de la información contable, se identifica a la nueva generación que necesita información confiable y requiere que se haga con rapidez, de acuerdo con Drucker (1987), el trabajo específico del gerente emprendedor de una empresa es hacer negocio empresa para ser más ágil y de calidad.

Según los datos disponibles en el sitio web de la CFC (2014), Brasil cuenta actualmente con más de 71.008 organizaciones/empresas contables y 515.011 profesionales registrados en el Consejo Federal de Contabilidad y estos profesionales y oficinas deben estar preparados para la automatización de procesos.

Teniendo el contador la función de guiar a los gerentes hacia el crecimiento de sus empresas, este profesional también juega un papel importante en la sociedad, y para ello, siempre debe estar en busca de actualizar sus conocimientos y comportarse éticamente, siendo el interlocutor del gobierno, con el contribuyente y la sociedad (CORONADO, 2017).

El contador que guía a sus clientes a contribuir correctamente, ayuda en el desarrollo del estado y con esto en el desarrollo del país. En este rol, la automatización es la clave para toda la atención del contador está en la generación de información y no en tareas aburridas y que consumen mucho tiempo de conferencias y lanzamientos. 
La competencia, profesionalidad, agilidad y especialización de los implicados en los procesos los hacen más coherentes, ágiles y funcionan correctamente. La práctica contable eficiente y especializada es el punto que se debe prestar atención al negocio perpetuado y son cada vez más eficientes y competitivos.

El profesional que entiende estos cambios de comportamiento que ha estado sucediendo, se especializa y actualiza, tendrá un papel importante y decisivo en la sociedad y las corporaciones. Todo el proceso depende de un ser humano, y esto, el ser humano tiene que tener las habilidades y destrezas para que el proceso automatizado funcione correctamente, además de un conocimiento sistémico de las empresas. La especialización y el conocimiento profundo son herramientas para este nuevo paradigma de cambio en la profesión contable.

\section{CONCLUSIÓN}

Como se indica en este artículo, la profesión contable desde el principio es importante para el desarrollo y crecimiento del país. La automatización de los procesos contables y el cambio de requisitos de la profesión harán que el profesional que hoy es visto como sólo el cumplimiento de los requisitos legales se extinga. Luego viene la oportunidad para que la profesión y los profesionales destaquen en otras áreas de sus conocimientos como la consultoría y la estrategia empresarial, poniendo en formación los vastos conocimientos adquiridos en educación.

Las oficinas contables contribuyen a la generación de puestos de trabajo, cumpliendo así su rol social, pero deben adaptarse a las nuevas dinámicas de los sistemas de información para que sean eficientes y produzcan con calidad y agilidad la información necesaria para las empresas y los gobiernos. Dado que la educación es una base para el desarrollo de cualquier nación, la mejora de la profesión y la educación del profesional implica una mejora significativa en la base de la sociedad y con ello eventos de mejora social y profesional.

El profesional contable ha dejado de ser el único que solo trae malas noticias a las empresas, con guías y cuentas por pagar, aportando una orientación estratégica a 
emprendedores y directivos, contribuyendo a que realicen sus funciones correctamente y con la agilidad que el mercado requiere a través de datos correctos y extraídos rápidamente.

Contador cuenta con todas las herramientas y conocimientos para automatizar la elaboración de estados financieros y lanzamientos, con el fin de aportar más agilidad y confiabilidad, emergiendo así como un aliado para que las empresas desempeñen su rol social con el crecimiento del negocio.

Las empresas que crezcan de forma sólida, inteligente y cumpliendo con sus obligaciones fiscales y corporativas con calidad y agilidad, un papel que el contador puede desempeñar junto con la gestión, harán que toda la sociedad se desarrolle. Si todos cumplen su papel con la economía y la sociedad, se generarán nuevos empleos, se pagarán más impuestos, disminuirán las tasas de pobreza y la oferta educativa será mayor.

La amenaza de la inexistencia de la profesión hará que los profesionales se reinventen y revisen su posición frente a las innovaciones. Aquellos que tienen una visión diferente de su posición como profesional de la contabilidad, combinando con las nuevas tecnologías de inteligencia artificial, automatización y especialización han ganado espacio en el mercado, diferente del profesional que es ajeno a los cambios y lucha contra ellos.

Por otro lado, es necesario mejorar la educación contable, para que las nuevas tecnologías se utilicen en la enseñanza y para que los alumnos salgan de las universidades preparadas para afrontar el mundo actual que requiere agilidad y calidad en la prestación de servicios.

Se observa una brecha a estudiar en cuanto a la distribución demográfica de los profesionales de la contabilidad, dado el grado de innovación de las empresas en determinadas regiones. Esto dará una visión más amplia de lo que puede o no puede ocurrir con la carrera. También se sugiere realizar estudios de caso en empresas innovadoras con un alto grado de automatización y el posicionamiento del profesional 
hacia esta empresa y sus resultados. Estos estudios contribuirán a una comprensión más amplia de lo que puede ocurrir en el futuro.

\section{REFERENCIAS}

ABREU, E. N. de. Regulamentação de auditoria: estudo da influência das fraudes contábeis de 2001. Goiânia: Universidade Federal de Goiás - UFG, 2013.

ACEVEDO, L. Business benefits of information technology. Chron, 2019. Disponível em: <http://smallbusiness.chron.com/business-benefits-informationtechnology-4021.html >. Acesso em: 10/mai/2021

ALLAHYARI, A.; RAMAZANI, M. Firm technological change and its effects on management accounting change: case study of Iranian manufacturing firms. Global Journal of Management and Business Research, v. 11, n. 9, 2011.

ALSHARAYRI, M. A. The E-Commerce impact on improving accounting information system in Jordanian Hotels. International Research Journal of Finance and Economics, v. 75, 2011.

ARAÚJO, F. M. de. A inteligência artificial e os seus impactos no mundo do trabalho. Fortaleza: Centro Universitário Fametro, 2020.

ARIBABA, F. et al. An evaluation of the impact of technological innovative entrepreneurial development programmes on the performance of small scale business in Nigeria. Global Journal of Business, Management And Accounting, v. 1, n. 1, 2011.

CARVALHOSA, M. de S. B. Comentários à lei de sociedades anônimas. Saraiva Educação SA, Editora: Saraiva Educação S. A., v. 1. 2017.

CFC. Conselho Federal de Contabilidade. Dados estatísticos do Conselho Federal de Contabilidade. 2014. Disponível em: http://www.cfc.org.br/. Acesso em: 12/mai/2021. 
CHIAVENATO, I. Empreendedorismo: dando asas ao espírito empreendedor. Editora manole, $4^{\mathrm{a}}$ ed. 2004.

CHOE, J. Impact of management accounting information and AMT on organizational performance. Journal of Information Technology, v. 19, 2004.

COELHO, J. M. A. Contabilidade: uma carreira em transformação. Disponível em: http://www.portalcfc.org.br/noticia.php?new=23196. Acesso em: 12/mai/2021.

CORDEIRO, C. M. R. Auditoria e Governança corporativa. Editora: IESDE, 2011.

CORONADO, O. Contabilidade gerencial básica. Editora: Saraiva Educação SA, 2017.

CUNHA, E. S. Oferta de crédito e emprego local nos municípios da região Nordeste: 2002-2015. Salvador: Federal da Bahia. 2019.

D'AURIA, F. Primeiros princípios de contabilidade pura. São Paulo: Departamento de Cultura e Ação Social, 1949.

DE OLIVEIRA REIS, A. et al. Perfil do profissional contábil: habilidades, competências e imagem simbólica. Revista Contemporânea de contabilidade, v. 12, n. 25, p. 95-116, 2015.

DINIZ, E. R. da S. et al. Governança eletrônica no Brasil e o papel das tecnologias da informação e comunicação. Florianópolis: Universidade Federal de Santa Catarina, 2014.

DOS SANTOS, M. J. A. et al. Um estudo comparativo entre o exame de suficiência do conselho federal de contabilidade, o exame nacional de desempenho de estudantes e a pós-graduação stricto sensu dos cursos de ciências contábeis do Estado do Rio de Janeiro. Polêm!ca, v. 13, n. 4, p. 1681-1698, 2014.

DRUCKER, P. F. Inovação e Espírito Empreendedor: prática e princípios. São Paulo: Pioneira, 1987. 
FAVERO, H. L. et al. Teoria e Prática. 4 ed. Editora: Atlas, São Paulo. 2006.

FIGUEIREDO, C. R.; STRASSBURG, U. A Contabilidade: aspectos históricos que influenciaram no seu desenvolvimento no decorrer do tempo. IX Jornada de Estudos Contábeis-UNIOESTE, 2008.

FILGUEIRA, J. M. et al. Um panorama estatístico de cunho econômico, social e educacional do estado do Rio Grande do Norte. HOLOS, v. 4, p. 198-215, 2010.

FURTADO, C. Dialética do Desenvolvimento. Rio de Janeiro, $2^{a}$ ed., Fundo de Cultura, 1964.

GIL, A. C. Métodos e técnicas de pesquisa social. 6. ed. São Paulo: Atlas, 2008.

GONDIM, A. MEl também tem obrigações fiscais. Contábeis, 2016. Disponível em: https://www.contabeis.com.br/noticias/26714/mei-tambem-tem-obrigacoes-fiscais/.

GRANDE, E. U. et al. The impact of accounting information systems (AIS) on performance measures: empirical evidence in Spanish SMEs. The International Journal of Digital Accounting Research, v. 11, 2011.

HEWLETT, S. A. Dilemas do desenvolvimento. Rio de Janeiro: Zahar, 1981.

IUDÍCIBUS, S. de; MARION, J. C. Introdução à teoria da contabilidade para o nível de graduação. 4 ed. São Paulo. Editora: Atlas, 2006.

JERMIAS, J.; GANI, L. Integrating business strategy, organizational configurations and management accounting systems with business unit effectiveness: a fitness landscape approach. Management Accounting Research, v. 15, 2004.

LEITE, E. F. O fenômeno do empreendedorismo. Saraiva Educação SA, 2017.

LEUSIN JÚNIOR, S. A inserção internacional de potências médias: evidências da afirmação de países em desenvolvimento a luz da evolução do sistema 
multilateral de comércio. Porto Alegre: Universidade Federal do Rio Grande do Sul, 2015.

MARCONI, M. de A.; LAKATOS, E. M. Metodologia do trabalho científico. São Paulo: Editora Atlas, 1992. 4a ed.

MAT, T. Z. Management accounting and organizational change: impact of alignment of management accounting system, structure and strategy on performance. Philosophy School of Accounting, Finance and Economics Faculty of Business and Law Edith Cowan University. Perth Western Austrália, 2010.

MENDES, M. Por que a economia do Brasil foi para o buraco? Brasil economia e governo, 2015. Disponível em: http://www.brasil-economiagoverno.org.br/2015/08/25/por-que-a-economia-brasileira-foi-para-o-buraco/. Acesso em: 14/mai/2021.

NETO, O. R. M. A profissionalização do contador no Brasil. São Paulo: Mackpesquisa, 2010.2 Disponível em: http://168.197.92.160/bitstream/handle/10899/14605/721_2008_0_15.pdf?sequence $=1$ \&isAllowed $=\mathrm{y} /$. Acesso em: 14/mai/2021.

NOGUEIRA, M. Práticas de contabilidade: relatório de estágio do Mestrado em Gestão. Coimbra: [s.n], 2010.

RAMALHO, T. C. L. Contabilidade criativa: a percepção dos Técnicos Oficiais de Contas. Lisboa: Instituto Superior de Contabilidade e Administração de Lisboa. 2015.

ROCHA, F. J. M. Educação e economia: uma abordagem sobre as consequências e condicionantes econômicas do desenvolvimento humano, com ênfase em educação. Brasília: Cadastro de Finanças Públicas, n. 5, 2004.

ROZENBAUM, S. Revolução digital deixa cinco profissões à beira da extinção. rfi, 2018. Disponível em: https://www.rfi.fr/br/economia/20180829-revolucao-digitaldeixa-cinco-profissoes-beira-da-extincao-diz-estudo. 
SANTOS, R. F. dos. Introdução à contabilidade: noções fundamentais. São Paulo: Saraiva, 2008.

SCOTT, W. The impact technology is having on the accounting profession. World conference on higher education, 2009.

SIEDENBERG, D. R. Dicionário do Desenvolvimento Regional. Santa Cruz: Edunisc, 2006.

SIMONS, R. Accounting control systems and business strategy: an empirical analysis. Accounting, Organizations and Society, v. 12,1987.

SOUZA, E. C. et al. As características qualitativas da informação contábil a partir da percepção dos profissionais de contabilidade. In: Congresso UFPE de Ciências Contábeis. 2018.

VASCONCELLOS, M. A. S. de. Economia Micro e Macro: Teoria e Exercícios, Glossário com 260 Principais Conceitos Econômicos. São Paulo: Atlas, 2000.

Enviado: Agosto de 2020.

Aprobado: Junio de 2021. 